\title{
SPATIALLY-AVERAGED DIFFUSIVITIES FOR POLLUTANT TRANSPORT IN VEGETATED FLOWS
}

\author{
JUN HUANG \\ Department of Civil \& Environmental Engineering, National University of Singapore, 1 Engineering Dr. 2 \\ Singapore 546900, Singapore \\ XIAOFENG ZHANG \\ Department of Civil \& Environmental Engineering, National University of Singapore, 1 Engineering Dr. 2 \\ Singapore 546900, Singapore \\ VIVIEN P. CHUA \\ Department of Civil \& Environmental Engineering, National University of Singapore, 1 Engineering Dr. 2 \\ Singapore 546900, Singapore \\ vivienchua@nus.edu.sg
}

Published 22 June 2016

\begin{abstract}
Vegetation in wetlands can create complicated flow patterns and may provide many environmental benefits including water purification, flood protection and shoreline stabilization. The interaction between vegetation and flow has significant impacts on the transport of pollutants, nutrients and sediments. In this paper, we investigate pollutant transport in vegetated flows using the Delft3DFLOW hydrodynamic software. The model simulates the transport of pollutants with the continuous release of a passive tracer at mid-depth and mid-width in the region where the flow is fully developed. The theoretical Gaussian plume profile is fitted to experimental data, and the lateral and vertical diffusivities are computed using the least squares method. In previous tracer studies conducted in the laboratory, the measurements were obtained at a single cross-section as experimental data is typically collected at one location. These diffusivities are then used to represent spatially-averaged values. With the numerical model, sensitivity analysis of lateral and vertical diffusivities along the longitudinal direction was performed at 8 cross-sections. Our results show that the lateral and vertical diffusivities increase with longitudinal distance from the injection point, due to the larger size of the dye cloud further downstream. A new method is proposed to compute diffusivities using a global minimum least squares method, which provides a more reliable estimate than the values obtained using the conventional method.
\end{abstract}

Keywords: Diffusion coefficient; numerical modeling; vegetated flow.

\section{Introduction}

Vegetation is commonly observed in rivers, wetlands and estuaries. Aquatic vegetation was historically considered only as a source of flow resistance, thus it was usually removed to enhance flow conveyance and reduce flooding during extreme flow

This is an Open Access article published by World Scientific Publishing Company. It is distributed under the terms of the Creative Commons Attribution 3.0 (CC-BY) License. Further distribution of this work is permitted, provided the original work is properly cited. 
conditions. In recent times, vegetation became known to provide ecological services that make it an integral part of river and coastal systems. In general, submerged vegetation leads to increased flow resistance, and the logarithmic velocity profiles in open-channel flows are altered. The interaction between vegetation and flow has significant impacts on the transport of pollutants, nutrients and sediments.

Empirical relationships for diffusivities, flow velocity and channel geometry have also been developed in vegetated flows. Earlier work in open-channel flows found that mixing processes are proportional to shear velocity and the characteristic length scale, i.e. water depth ${ }^{1,2}$. The presence of vegetation changes the velocity and length scales in the flow, thereby leading to changes in the diffusivities. Ref. 3 examined the effect of vegetation on flow diffusivities and found that they are proportional to the mean flow velocity and the stem diameter. These values are comparable for all vegetation densities under similar flow conditions.

The objective of this study is to understand the diffusion rate in vegetated and nonvegetated flows, and to propose a new method to compute the spatially-averaged diffusivities in both types of flow.

\section{Numerical Model}

\subsection{Delft3D model}

The Delft3D-FLOW model coupled with a vegetation module is employed to perform the numerical simulations. The governing equations are the three-dimensional, Reynoldsaveraged Navier-Stokes equations under the Boussinesq approximation and hydrostatic assumption. The transport of salinity, heat and passive tracers are described with the advection-diffusion equations. The eddy viscosity and diffusivity terms are computed with the $k-\varepsilon$ turbulence closure model. The bottom friction is computed by specifying the Chezy coefficient.

A vegetation module is developed to take into account the effect of vegetation on flow $^{4}$. The vegetation is represented as vertical cylinders with pre-defined density, height and diameter. Additional terms are introduced in the momentum equations and the turbulence closure model to account for the resistance of the vertical cylinders.

In the momentum equations, an additional source term is added to simulate the effect of friction force caused by cylinders,

$$
\begin{aligned}
& F(u)=\frac{1}{2} C_{D} m(z) d(z) u \sqrt{u^{2}+v^{2}} \\
& F(v)=\frac{1}{2} C_{D} m(z) d(z) v \sqrt{u^{2}+v^{2}}
\end{aligned}
$$

where $C_{D}$ is the drag coefficient, $d(z)$ is the stem diameter, and $m(z)$ is the stem density. 
In the turbulence model, two source terms are added,

$$
\begin{gathered}
\left(\frac{\partial k}{\partial t}\right)_{\text {vegetation }}=\frac{1}{1-\lambda(z)} \frac{\partial}{\partial z}\left((1-\lambda(z))\left(v+\frac{v_{v}}{\sigma_{k}}\right) \frac{\partial k}{\partial z}\right)+T(z) \\
\left(\frac{\partial \varepsilon}{\partial t}\right)_{\text {vegetation }}=\frac{1}{1-\lambda(z)} \frac{\partial}{\partial z}\left((1-\lambda(z))\left(v+\frac{v_{v}}{\sigma_{\varepsilon}}\right) \frac{\partial \varepsilon}{\partial z}\right)+c_{2 \varepsilon} \frac{T(z)}{\tau_{\text {eff }}}
\end{gathered}
$$

where $\lambda(z)$ is the horizontal cross-section area of the cylinder structure per unit area at height $z$ and is given by,

$$
\lambda(z)=\frac{\pi}{4} D(z)^{2} m(z)
$$

$T(z)$ is a turbulence source generated by rigid cylinders and is described as,

$$
T(z)=F \sqrt{u^{2}+v^{2}}
$$

The second term on the right side of Eq. (4) describes the dissipation rate of the turbulence generated by rigid cylinders. $\tau_{e f f}$ is the rate of turbulent kinetic energy production by the cylinders and $C_{2 \varepsilon}$ is the coefficient of dissipation rate.

\subsection{Model setup}

The model domain is a narrow and straight rectangular channel with $28 \mathrm{~m}$ length, $0.6 \mathrm{~m}$ width and $0.5 \mathrm{~m}$ depth (Fig. 1). The horizontal Cartesian grid resolution was set as $\Delta x=$ $0.15 \mathrm{~m}, \Delta y=0.055 \mathrm{~m}$. In the vertical, the model has $20 \sigma$-layers. The resolution increases towards the bottom to allow better representation of the bottom vegetation. Three meters of gravel bottom is placed immediately downstream of the inlet. The vegetation patch in the numerical model is $22 \mathrm{~m}$ long and placed downstream of the gravel area. The vegetation module requires that the bulk drag coefficient $C_{D}$, characteristics of vegetation (height $h_{v}$ and diameter $d$ ), density of vegetation population $m$, and turbulence length scale coefficient $c_{l}$ are specified. The height, diameter and density of vegetation are $h_{v}=$ $0.08 \mathrm{~m}, d=0.006 \mathrm{~m}$ and $m=400 / \mathrm{m}^{2}$, respectively. The turbulence length scale coefficient is $c_{l}=0.8$. The bulk drag coefficient is set as $C_{D}=1.2$.

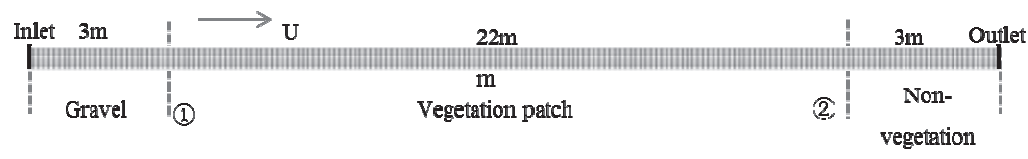

Fig. 1. Computational grid for laboratory flume set-up. 
The simulation is initialized with a flat free surface and a quiescent velocity field. There are two open boundaries in the model, located at the upstream and downstream ends. At the inlet (upstream end), the flow rate is specified and set to be similar to the flow rate used in the flume experiment. At the outlet (downstream end), the water surface elevation is specified and set to zero.

The model is calibrated and validated using experimental data under unidirectional flow. The model-predicted velocity and turbulent kinetic energy (TKE) at several locations in the longitudinal direction are compared with experimental data obtained using an array of rigid rods as submerged vegetation [5]. The user-defined background eddy viscosities and diffusivities are adjusted to match the model-predicted profiles with experimental data. The user-defined horizontal and vertical eddy viscosities are set as $v_{H}^{\text {back }}=0.8 \mathrm{~m}^{2} / \mathrm{s}$ and $v_{V}^{\text {back }}=1 \times 10^{-6} \mathrm{~m}^{2} / \mathrm{s}$ respectively. The user-defined horizontal and vertical eddy diffusivities are set as $D_{H}{ }^{\text {back }}=9.2 \times 10^{-5} \mathrm{~m}^{2} / \mathrm{s}$ and $D_{V}{ }^{\text {back }}=4.4 \times 10^{-5} \mathrm{~m}^{2} / \mathrm{s}$, respectively. Note that the user-defined horizontal and vertical eddy diffusivities only comprise one part of the total diffusivity.

\subsection{Model simulations}

The model simulates the transport of pollutants with the introduction of a passive tracer. A continuous release of passive tracer was made at the mid-depth and mid-width of the flume. The tracer injection is made in the region where the flow is fully developed, i.e. at the longitudinal position $8 \mathrm{~m}$ from the start of the vegetation patch. The location of injection is defined as $x=0 \mathrm{~m}$. The injection rate was matched with the ambient flow to avoid jet-induced mixing. The tracer has a concentration of $10 \mathrm{~kg} / \mathrm{m}^{3}$.

\section{Results}

The model is used to compute the lateral and vertical diffusion coefficients. These values are subsequently used to determine the time required for the tracer to reach the Fickian dispersive regime.

\subsection{Lateral and vertical diffusivities}

The lateral and vertical diffusivities were estimated by fitting the predicted concentration profile to the theoretical Gaussian plume profile (Nepf 1997). The concentration $C$ at location $(x, y, \mathrm{z})$ is:

$$
C(x, y, z)=\frac{\dot{M}}{4 \pi x \sqrt{D_{y} D_{z}}} \exp \left(-\frac{y^{2} \bar{u}}{4 D_{y} x}-\frac{z^{2} \bar{u}}{4 D_{z} x}\right)
$$

where $\dot{M}$ is the dye mass injection rate at $(0,0,0)$ and $\bar{u}$ is the cross-sectional averaged velocity. The lateral diffusivity $D_{y}$ was obtained via the least squares method, i.e. by minimizing: 


$$
\varepsilon_{r m s}=\sqrt{\frac{1}{N} \sum_{\forall y}\left[\left(\frac{C(y)}{C_{\max }}\right)-\exp \left(\frac{y^{2} \bar{u}}{4 D_{y} x}\right)\right]^{2}}
$$

where $C(y)$ is the concentration distribution in the lateral direction at a cross-section, $C_{\max }$ represents the maximum concentration and $N$ is the total number of data points. Similarly, the vertical diffusivity $D_{z}$ was obtained via the least squares method, i.e. by minimizing:

$$
\varepsilon_{r m s}=\sqrt{\frac{1}{N} \sum_{\forall y}\left[\left(\frac{C(\mathrm{z})}{C_{\max }}\right)_{\text {predicted }}-\exp \left(\frac{z^{2} \bar{u}}{4 D_{z} x}\right)\right]^{2}}
$$

where $C(z)$ is the concentration distribution in the vertical direction at a cross-section.

\subsection{Sensitivity analysis of lateral and vertical diffusivities}

Sensitivity analysis of lateral and vertical eddy diffusivities was performed at 8 crosssections, from $\mathrm{x}=1$ to $8 \mathrm{~m}$ downstream of the point dye source. The least squares method in Eqs. (8) and (9) were used to compute the lateral and vertical eddy diffusivities at every single cross-section. For each case, we plot the curve $\varepsilon=\mathrm{f}\left(D_{y}\right)$ (or $\left.\varepsilon=\mathrm{f}\left(D_{y}\right)\right)$ and the minimum value of each curve is selected as the lateral and vertical diffusivities at a particular longitudinal position.

Figs. 2-5 show the results of the sensitivity analysis. The lateral and vertical diffusivities increase with longitudinal distance from the injection point, which may be due to the larger size of the dye cloud further downstream. Furthermore, the magnitude and rate of increase is significantly larger in vegetated compared to non-vegetated flow. The turbulence kinetic energy is increased significantly in the presence of vegetation, which results in an increase in the magnitude and rate of increase of diffusivities.

The diffusivities are calculated at a single cross-section for experimental studies, as data is typically collected at one location. These diffusivities are used to represent spatially-averaged values. However, this method over- or under-estimates the spatiallyaveraged diffusivity since the diffusivity changes with longitudinal position (Figs. 2-5). A new method is proposed to compute diffusivities using a global minimum least square method. 

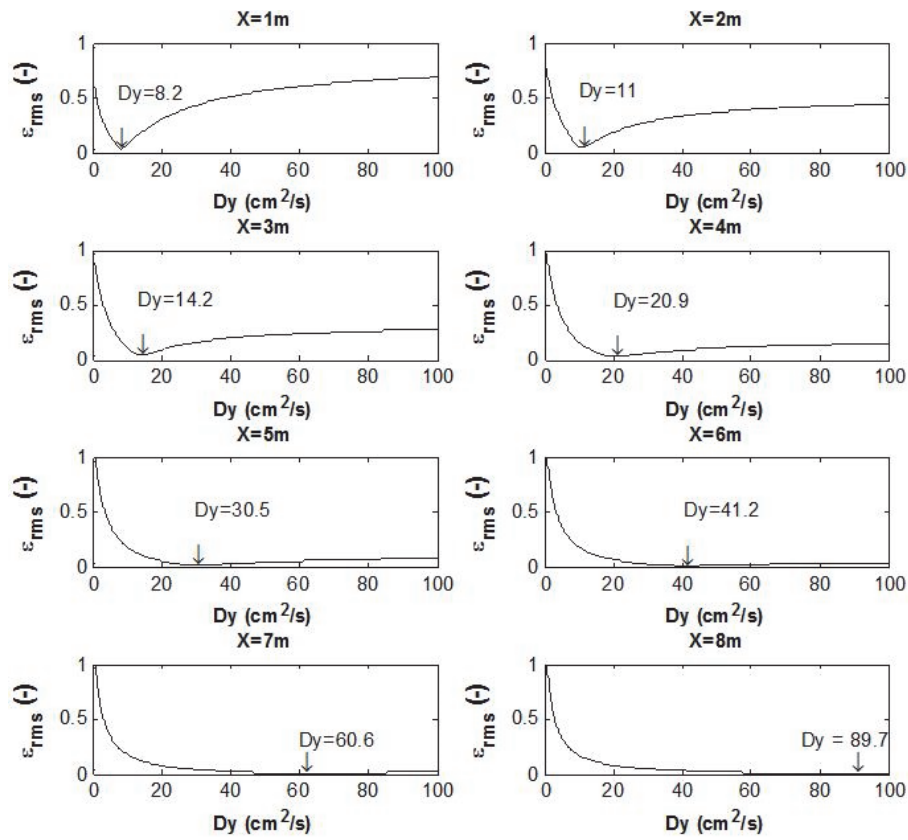

Fig. 2. Sensitivity analysis of lateral diffusion coefficient in vegetated flows.
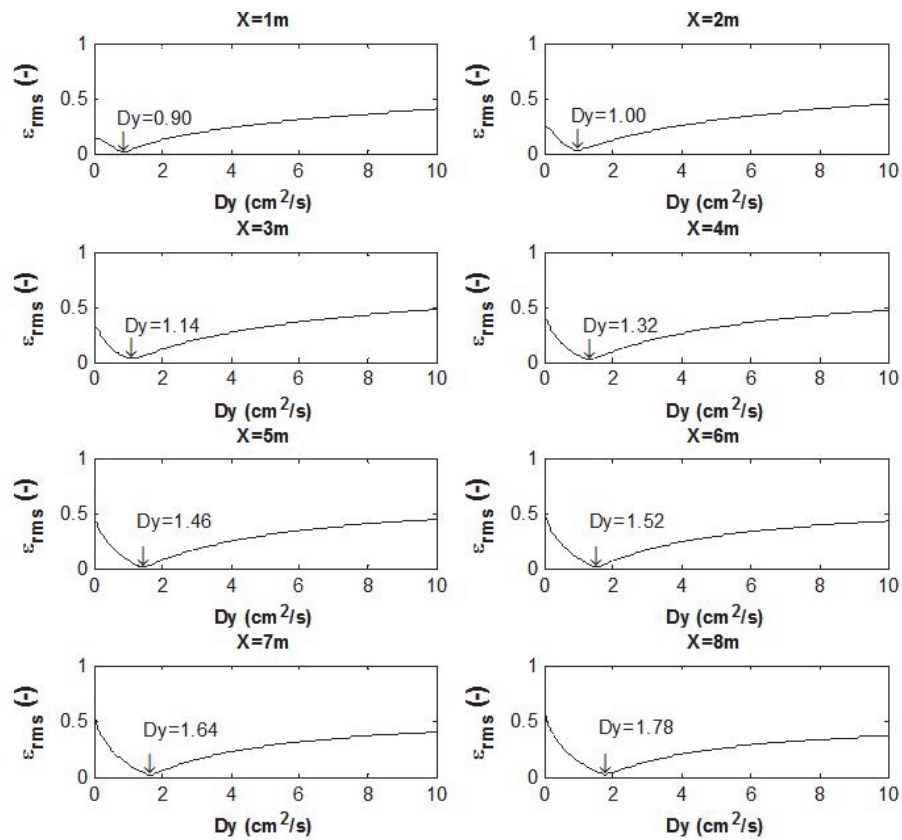

Fig. 3. Sensitivity analysis of lateral diffusion coefficient in non-vegetated flows. 

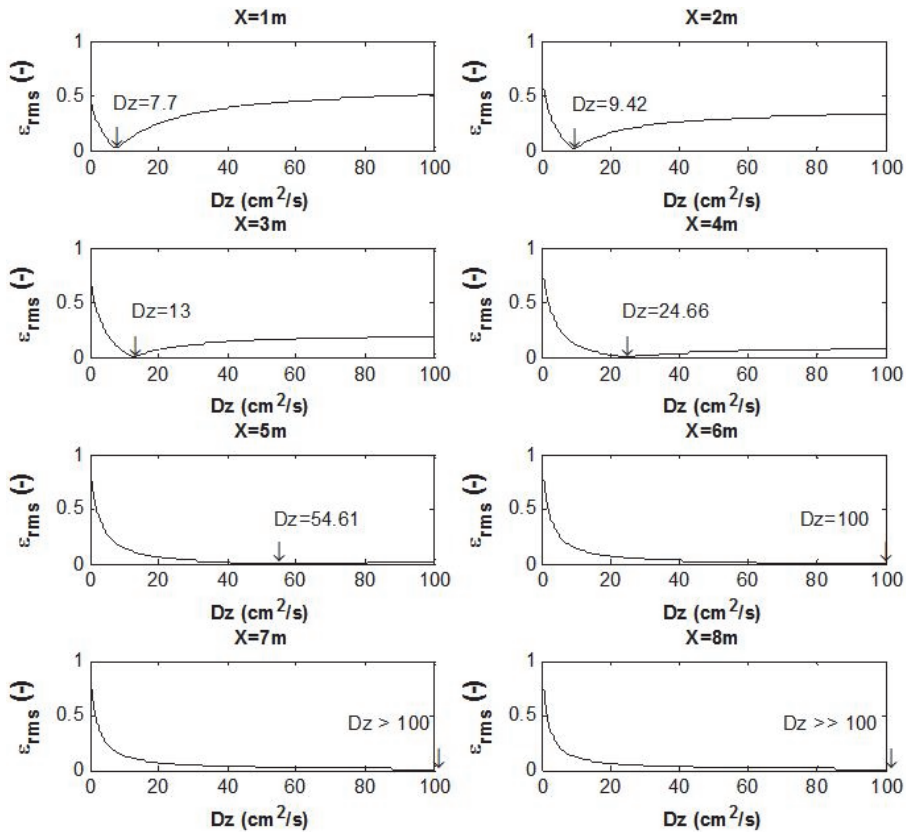

Fig. 4. Sensitivity analysis of vertical diffusion coefficient in vegetated flows.
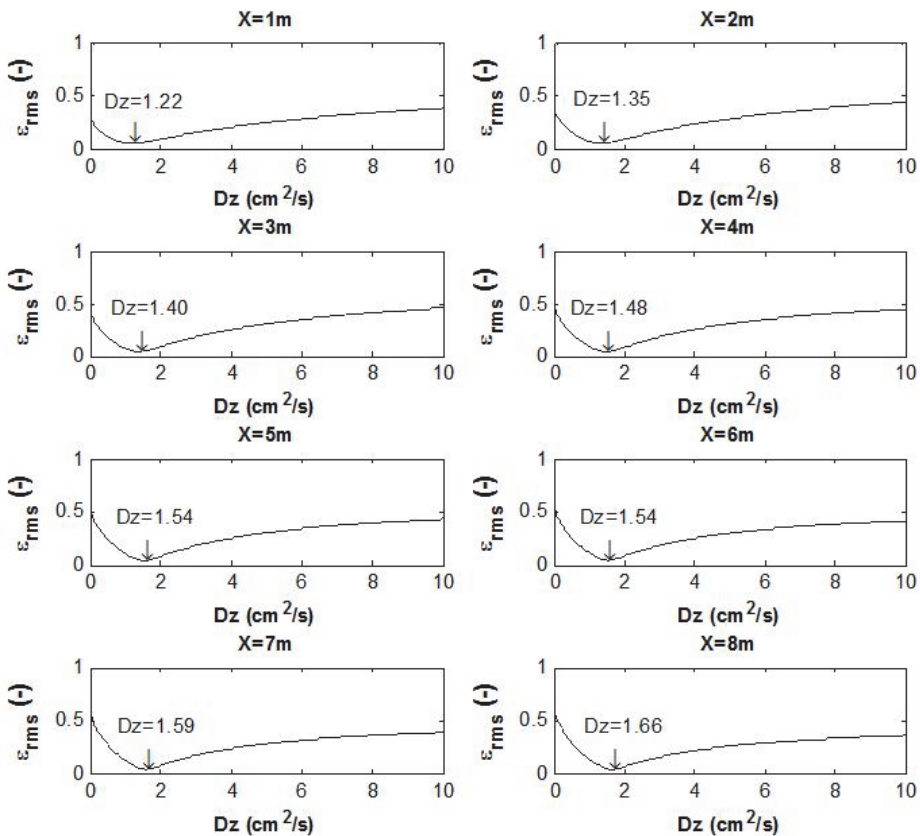

Fig. 5. Sensitivity analysis of vertical diffusion coefficient in non-vegetated flows. 
In a similar manner, $\varepsilon_{r m s}$ is computed at cross-sections spaced $1 \mathrm{~m}$ apart from $\mathrm{x}=1$ to $8 \mathrm{~m}$ using Eqs. (8) and (9). Next, we average the $\varepsilon_{r m s}$ values of the 8 cross-sections and plot the curve of $\varepsilon_{g l o b a l}$ against $D_{y}$ and $D_{z}$. The lateral and vertical diffusivities are determined at the minimum value of the $\varepsilon_{\text {global }}$ curves. The variation of $\varepsilon_{g l o b a l}$ at 8 crosssections against against $D_{y}$ and $D_{z}$ for different flow conditions are presented in Figs. 6 and 7 . The diffusivity values calculated with the global minimum least square method are summarized in Table 1. We found that this method provides a more reliable estimate than the values obtained using the conventional method. The lateral and vertical diffusivities have the same order of magnitude since the channel width and depth are comparable. The diffusivities are an order of magnitude larger in vegetated flow compared to nonvegetated flows.
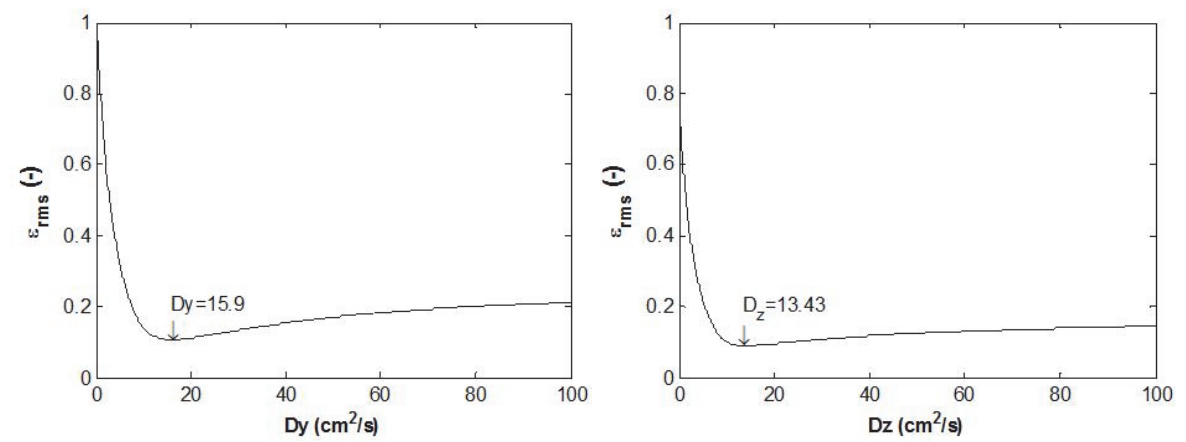

Fig. 6. Lateral and vertical diffusivities in vegetated flows computed with the global minimum least squares method.
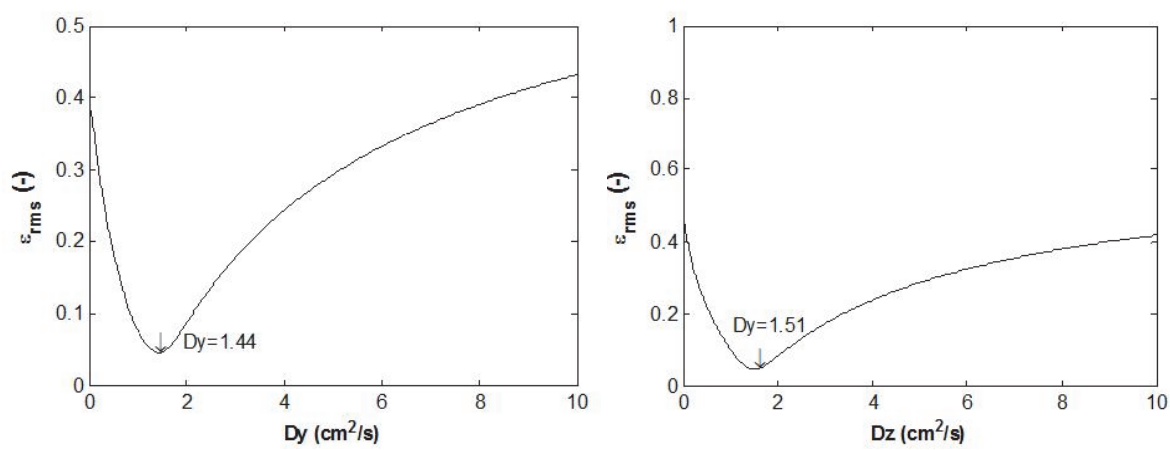

Fig. 7. Lateral and vertical diffusivities in non-vegetated flows computed with the global minimum least squares method. 
Table 1. Lateral and vertical diffusivities determined by global minimum least squares method.

\begin{tabular}{lll}
\hline Vegetation & Diffusivities & Value \\
\hline No & $D_{y}$ & 1.44 \\
No & $D_{z}$ & 1.51 \\
Yes & $D_{y}$ & 15.90 \\
Yes & $D_{z}$ & 13.43 \\
\hline
\end{tabular}

\section{Conclusions}

In this study, we aim to obtain an improved understanding of diffusivities in vegetated and non-vegetated flows. The diffusion coefficients are estimated by fitting the modelpredicted concentration profile to the theoretical concentration profile. The modelpredicted lateral and vertical diffusivities are on the same order of magnitude, while diffusivities are an order of magnitude larger in vegetated compared to non-vegetated flow. Sensitivity analysis of lateral and vertical diffusivities show that diffusivities increase with longitudinal distance from the injection point. Hence, a global minimum least square method is proposed to compute diffusivities, which provides a more reliable estimate than the values obtained using the conventional method at a single cross-section.

\section{Acknowledgments}

J.H. acknowledges the support of the Singapore Delft Water Alliance M.S. scholarship. X.Z. acknowledges the support of NUS Research Scholarship. The authors acknowledge the support of the National University of Singapore research grants (WBS R-302-00-068133, R-302-000-072-112).

\section{References}

1. H. B. Fischer, Mixing in Inland and Coastal Waters. (Academic Press, New York, 1979)

2. J. W. Elder, J. Fluid Mech., 5, 544 (1959)

3. E. T. Alvin, A field investigation of diffusion within a submerged plant canopy. (Master Thesis, MIT, USA, 1997)

4. S. Temmerman, T. J. Bouma, G. Govers, Z. B. Wang, M. B. De Vries and P. M. J. Herman, J. Geophys. Res.: Earth Surf., 110 (2005).

5. X. Zhang, J. H. F. Cheong and V. P. Chua, Numerical simulations of flow over mangrove roots, In Proc. $7^{\text {th }}$ Intl. Symp. Environ. Hydraul., Singapore. 